
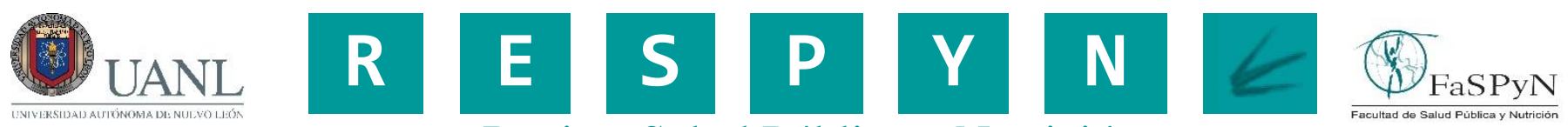

Revista Salud Pública y Nutrición

\title{
PATRONES DE ALIMENTOS Y SU RELACIÓN CON EL RIESGO DE PRESENTAR DEPRESIÓN EN ASPIRANTES UNIVERSITARIOS DE NUEVO INGRESO.
}

\author{
FOOD PATTERNS AND THEIR RELATION TO THE RISK OF PRESENTING DEPRESSION IN NEW ENTRY \\ UNIVERSITY APPLICANTS
}

\section{Olvera Castillo Ma. del Carmen ${ }^{1}$, Palos Lucio Ana Gabriela ${ }^{1}$, Aradillas García Celia², Padrón Salas Aldanely ${ }^{3}$, Sánchez-Armáss Cappello Omar ${ }^{4}$, Olvera León Guadalupe del Carmen ${ }^{4}$.}

1 Facultad de Enfermería y Nutrición, Universidad Autónoma de San Luis Potosí, México. 2 Centro de Investigación Aplicada en Ambiente y Salud, Universidad Autónoma de San Luis Potosí, México. 3 Facultad de Medicina, Universidad Autónoma de San Luis Potosí, México. 4 Facultad de Psicología, Universidad Autónoma de San Luis Potosí, México.

Citation: Olvera Castillo MC., Palos Lucio AG., Aradillas García C., Padrón Salas A., Sánchez-Armáss CO., Olvera León GC. (2018) Patrones de alimentos y su relación con el riesgo de presentar depresión en aspirantes universitarios de nuevo ingreso. Revista de Salud Pública y Nutrición, 17(2), 1-10

Editor: Esteban G. Ramos Peña, Dr. CS., Universidad Autónoma de Nuevo León, Facultad de Salud Pública, Monterrey Nuevo León, México.

Copyright: (C2018 Olvera Castillo MC et al. This is an open-access article distributed under the terms of Creative Commons Attribution License [CC BY 4.0], which permits unrestricted use, distribution, and reproduction in any medium, provided the original author and source are credited.

Competing interests: The authors have declared that no competing interests exist.

DOI: https://doi.org/10.29105/respyn17.2-1

Recibido: 7 de abril 2018;

Aceptado: 20 de junio 2018

Email: nutriolvera@gmail.com 


\title{
PATRONES DE ALIMENTOS Y SU RELACIÓN CON EL RIESGO DE PRESENTAR DEPRESIÓN EN ASPIRANTES UNIVERSITARIOS DE NUEVO INGRESO
}

\author{
Olvera Castillo Ma. del Carmen ${ }^{1}$, Palos Lucio Ana Gabriela ${ }^{1}$, Aradillas García Celia ${ }^{2}$, Padrón Salas Aldanely ${ }^{3}$, \\ Sánchez-Armáss Cappello Omar ${ }^{4}$, Olvera León Guadalupe del Carmen ${ }^{4}$.
}

\begin{abstract}
1 Facultad de Enfermería y Nutrición, Universidad Autónoma de San Luis Potosí. 2 Centro de Investigación Aplicada en Ambiente y Salud, Universidad Autónoma de San Luis Potosí. 3 Facultad de Medicina, Universidad Autónoma de San Luis Potosí. 4 Facultad de Psicología, Universidad Autónoma de San Luis Potosí.
\end{abstract}

\begin{abstract}
RESUMEN
Introducción: La universidad es una experiencia estresante, los estudiantes modifican sus patrones de alimentos como una respuesta de adaptación, siendo que esto podría aumentar el riesgo de que generen sintomatología relacionada con la depresión. Objetivo: Identificar la relación entre el patrón de alimentos de los aspirantes universitarios y el riesgo de presentar depresión. Material y Métodos: Estudio transversal, se analizó la información de 1556 aspirantes universitarios de entre 18 y 25 años de edad que participaron en la investigación multidisciplinaria en obesidad y factores socio-ambientales ("UP AMIGOS 2008"). Se les aplicó un cuestionario sobre síntomas depresivos (CESD-10) y un cuestionario basado en el índice de alimentación saludable. Para conocer la diferencia de medias entre muestras categóricas se realizó prueba de chi cuadrada. La comparación de datos se realizó mediante un análisis de regresión logística. Resultados: Se encontró que existe una relación positiva entre el riesgo de desarrollar depresión con el consumo elevado de embutidos (salchicha, jamón, chorizo) $(P=0.008)$, el bajo consumo de carnes $(P=0.013)$ y con pertenecer al sexo femenino $(P<0.001)$. Conclusiones: Los síntomas depresivos deben monitorearse en los universitarios en especial las mujeres y en aquéllos que consumen alimentos con alto contenido de sal.

Palabras Clave: Patrón de alimentos, depresión, aspirantes universitarios.
\end{abstract}

\section{ABSTRACT}

Introduction: The university is a stressful experience; students modify their food patterns as an adaptation response that can increase the risk of generating the disease symptomatology related to depression. Objective: To identify the relationship between the food pattern of college students and the risk of developing depression. Methods: Cross-sectional study, analyzed data from 1556 university students aged 18-25 years old who participated in multidisciplinary research on obesity and socioenvironmental factors ("UP AMIGOS 2008"). They answered the questionnaire on depressive symptoms (CESD-10) and a questionnaire based on the healthy eating index. To know the difference of means between categorical samples, chi square test was performed. Data were compared using logistic regression analysis. Results It was found that there is a positive relationship between the risk of developing depression with high intake of cold meat (sausage, ham, chorizo) $(P=0.008)$, low meat intake $(P=0.013)$ and belong to the female sex $(P<0.001)$. Conclusions: Depressive symptoms should be monitored in college students, especially women, and in those who consume foods that are high in salt.

Key words: Food patterns, depression, university aspirants 


\section{Introducción}

Un patrón de alimentos se puede definir como la distribución de alimentos por frecuencia y/o cantidad en la dieta habitual. Los patrones de alimentos seguidos por un individuo están basados fundamentalmente en el estilo de vida. La configuración de un estilo depende de la forma de pensar, sentir y actuar, las condiciones de vida y de los factores socioculturales de una persona (ÁlvarezMuñárriz \& Álvarez de Luis, 2009).

Cuando los patrones de alimentos son inadecuados tendrán repercusiones tanto físicas como mentales. En el área física aunado a la genética, contribuirán en el desarrollo de enfermedades crónico-degenerativas como obesidad, hipertensión o diabetes. En tanto que en el ámbito mental los hábitos alimentarios tienen efectos en los estados de ánimo, e independientemente de la razón subyacente del trastorno, estos patrones afectarán los sentimientos y de manera viceversa (El Ansari, Adetunji \& Oskrochi, 2014). Diversos estudios documentan que una inadecuada nutrición y una pobre calidad de la dieta (comida rápida, productos de origen animal, confitería) están frecuentemente relacionados con problemas mentales (Jacka et al., 2010). En específico, el estudio de la relación entre patrones de alimentación y depresión ha cobrado suma importancia.

Según datos de la Encuesta Nacional sobre el Uso de Drogas y la Salud (NSDUH), por sus siglas en inglés, se estima que 16,1 millones de adultos mayores de 18 años en los Estados Unidos tuvieron al menos un episodio depresivo mayor en el último año ("Major Depression”, 2017). En estudiantes universitarios existen estudios como el llevado a cabo por Arrivillaga-Quintero, Cortés-García, GoicocheaJiménez \& Lozano-Ortiz (2004) de corte trasversal en donde en una universidad de Colombia, la prevalencia de depresión fue de $30 \%$. Por otro lado, Riveros, Hernández \& Rivera (2014) describió la prevalencia de esta enfermedad en estudiantes de una universidad en Lima, Perú donde el 9.7\% padece depresión leve, $2.8 \%$ depresión moderada y $0.05 \%$ depresión severa. Así mismo en población mexicana Álvarez-Gasca, Guillén-Cadena, Ríos-Saldaña y Jiménez-Martínez (2011) refieren que los estudiantes de la Universidad Autónoma de México el 33\% presentó depresión baja, $18.6 \%$ moderada, $23.6 \%$ depresión severa y $24.7 \%$ depresión alta.
Probablemente estas diferencias están relacionadas con el tipo de instrumentos de medición utilizados.

Las consecuencias de padecer depresión las reporta Hysenbegasi, Hass \& Rowland (2005) donde encontró que los estudiantes que padecían esta enfermedad mental indicaban haber perdido un número significativo de clases y exámenes, habían descartado cursos y faltaban a actividades sociales. En cuanto al rendimiento académico Fouilloux et al. (2013) reporta que en los estudiantes de primer año de la licenciatura de Médico Cirujano el 23\% presentaba síntomas depresivos, dentro de estos; el $68 \%$ reprobaron alguna materia, lo que supuso un mayor riesgo de reprobación de 2.4 veces, comparados con quienes no tenían dicha sintomatología. Asimismo, encontraron que el promedio de calificaciones de los estudiantes con sintomatología depresiva fue significativamente menor $(\mathrm{p}<0.05)$ que el promedio de los estudiantes sin síntomas.

En cuanto al consumo de alimentos y depresión, Appleton et al. (2007) indican que un bajo consumo de pescado (omega 3) está relacionado con la depresión, sin embargo, hay estudios prospectivos que no apoyan dicha asociación como el llevado a cabo por Hakkarainen et al. (2004).

Además, en un estudio transversal se observó asociación entre los síntomas depresivos con un consumo menos frecuente de frutas / verduras y carne (Mikolajczyk, El Ansari \& Maxwell, 2009), mientras que otros estudios muestran menores probabilidades de depresión con un alto consumo de carne (Meyer et al., 2013). Los estudios de la relación entre el consumo de nutrientes aislados y la depresión han sido inadecuados y producidos resultados inconsistentes debido a que han fallado en considerar las complejas interacciones entre los nutrientes (Martínez-González \& Sánchez-Villegas, 2016). Es por eso, que investigar la asociación entre patrones de alimentos y el riesgo de presentar depresión provee un mejor entendimiento de dicha asociación (Oddy et al., 2009).

El rol potencial del consumo de alimentos en el desarrollo de la depresión y su consecuente prevención es un área que debe ser estudiada, por lo que la presente investigación trata de determinar si existe relación entre el patrón de alimentos de los 
aspirantes universitarios y el riesgo de presentar depresión.

\section{Material y Métodos}

Fue un estudio de corte transversal. El universo de estudio fueron los aspirantes universitarios para ingresar al ciclo escolar comprendido entre 20082013 en la Universidad Autónoma de San Luis Potosí. La información de los participantes fue obtenida de las bases de datos pertenecientes a la investigación UP AMIGOS 2008 (Universidades de San Luis Potosí e Illinois: Una Investigación Multidisciplinar sobre Obesidad y Factores socioambientales). Esta base de datos ya ha sido utilizada previamente en otros estudios por Wiley et al., 2011; Raffaellil et al., 2013; Olvera-Castillo, Palos-Lucio, Aradillas-García \& Padrón-Salas, 2018. La muestra de estudio correspondió aquellos aspirantes que cumplían con los criterios. Se incluyeron aspirantes entre 18 y 25 años que además de realizarse el examen médico como requerimiento para el ingreso, contestaron el cuestionario CESD-10 y el cuestionario basado en el Índice de Alimentación Saludable. Se excluyeron aquellos aspirantes que se encontraban bajo un régimen específico de alimentación o presentaban previamente un diagnóstico de enfermedad mental.

\section{Instrumentos}

Los aspirantes contestaron un cuestionario de frecuencia de consumo de alimentos. Esta herramienta está basada en el Índice de Alimentación Saludable (IAS) (Navarro-Norte \& Moncada-Ortiz, 2011) este a su vez está basado en el índice de alimentación saludable (HEI, por sus siglas en inglés) norteamericano (Kennedy, Ohls, Carlso \& Fleming, 1995). La herramienta en su forma original contiene 10 variables que representan los grupos de alimentos: 1- Cereales y derivados, 2-Verduras, 3Frutas, 4-Leche y derivados, 5-Carnes, 6Legumbres, 7-Embutidos y fiambres, 8-Dulces, 9Refrescos con azúcar y 10-Variedad de la dieta. Para este estudio solamente se consideraron las variables 1 a 9. Debido a que el instrumento fue elaborado bajo guías alimentarias propias de otro país; la variable 10 hacía referencia a si la persona cumplía o no con las recomendaciones alimenticias; se consideró que habría un margen de error al indicar un consumo semanal o diario diferentes al de México. El puntaje no fue modificado debido a que la variable que fue eliminada solo otorgaba 1 o 2 puntos dependiendo si se cumplía la recomendación alimentaria. Cada una de las variables se divide a su vez en 5 categorías que hacen referencia a la frecuencia de consumo: 1consumo diario, 2-tres o más veces a la semana, pero no diario, 3-una o dos veces a la semana, 4-menos de una vez por semana, 5- nunca o casi nunca. Cada opción de respuesta tiene un puntaje, al sumarse se obtienen 3 posibles puntuaciones que hacen referencia a la calidad de la alimentación: alimentación saludable: > 80 puntos, necesita cambios: >50-80 puntos y poco saludable < 50 puntos (Navarro-Norte y Moncada-Ortiz, 2011).

Con respecto a los síntomas depresivos, los encuestados completaron la versión de 10 ítems del Center for Epidemiological Studies Depression Scale (CESD-10) (Kohout, Berkman, Evans \& CornoniHuntley, 1993). En un estudio llevado a cabo en población mexicana se comparó el CESD-20 y CESD-10, ambas versiones tenían buenas propiedades psicométricas (Bojorquez-Chapela \& Salgado de Snyder, 2009). En este instrumento se debe calificar la frecuencia con la que se experimenta cada síntoma durante la semana pasada con cuatro opciones de respuesta, rara vez o ninguna de las veces (menos de 1 día), alguna vez o una pequeña parte del tiempo (1-2 días), ocasionalmente o una cantidad moderada de tiempo y todo el tiempo (5-7 días). Las puntuaciones posibles van de 0 a 30 ; una puntuación de 10 o más se considera positivo para la presencia de depresión.

El estudio fue aprobado por el Comité de Bioética e Investigación para la Salud de la Secretaria de Salud del gobierno del estado de San Luis Potosí y por el Comité de Ética en investigación de la Facultad de Enfermería y Nutrición de la UASLP.

El estudio se realizó con la información brindada por los pacientes con previo consentimiento informado.

\section{Análisis de datos}

Se llevó a cabo un análisis descriptivo de las variables cuantitativas mediante medidas de tendencia central y dispersión. Para conocer la diferencia de medias entre muestras categóricas se realizó prueba de chi cuadrada. La comparación de datos entre los grupos se realizó mediante un análisis de regresión logística.

Los datos fueron analizados mediante el paquete estadístico STATA v.11 


\section{Resultados}

La población de estudio estuvo conformada por 1556 aspirantes, de los cuales un poco más de la mitad $(54.78 \%)$ fueron mujeres. La edad de los participantes estuvo comprendida entre 18 y 25 años con una media de edad de $18.84 \pm 1.30$ años en mujeres y $19.16 \pm 1.53$ años en hombres.

Según el IMC, hubo mayor prevalencia de normopeso con un $60.87 \%$, en segundo lugar, sobrepeso con un $21.81 \%$, seguido de obesidad grado 1 con un $7.63 \%$, cuarto lugar bajo peso con un $7.5 \%$ y para el penúltimo y último lugar obesidad grado 2 con $1.67 \%$ y obesidad grado 3 con un $0.51 \%$. La distribución del grupo de estudio según sexo, edad y estado nutricional se describen en la Tabla 1.

\begin{tabular}{|c|c|c|c|c|}
\hline Variable & $\begin{array}{c}\text { Población } \\
\text { general } \\
n=1556 \\
N\end{array}$ & $\begin{array}{c}\text { Con sintomatología } \\
\text { depresiva } \\
n=104 \\
N\end{array}$ & $\begin{array}{c}\text { Sin sintomatología } \\
\text { depresiva } \\
n=1452 \\
N\end{array}$ & $\begin{array}{l}\text { Valor } \\
\text { de } P\end{array}$ \\
\hline \multicolumn{5}{|l|}{ Edad } \\
\hline 18 & 806 & 54 & 752 & \multirow{8}{*}{0.816} \\
\hline 19 & 364 & 25 & 339 & \\
\hline 20 & 198 & 13 & 185 & \\
\hline 21 & 86 & 4 & 82 & \\
\hline 22 & 37 & 0 & 37 & \\
\hline 23 & 30 & 5 & 25 & \\
\hline 24 & 24 & 3 & 21 & \\
\hline 25 & 11 & 0 & 11 & \\
\hline \multicolumn{5}{|l|}{ Sexo } \\
\hline Mujeres & 851 & 79 & 772 & \multirow{2}{*}{$<0.001$} \\
\hline Hombres & 705 & 25 & 680 & \\
\hline \multicolumn{5}{|l|}{ IMC[1] } \\
\hline $\begin{array}{l}\text { Bajo peso } \\
\left(<18.4 \mathrm{~kg} / \mathrm{m}^{2}\right)\end{array}$ & 117 & 7 & 110 & 0.588 \\
\hline $\begin{array}{l}\text { Normopeso } \\
\left(18.5-24.9 \mathrm{~kg} / \mathrm{m}^{2}\right)\end{array}$ & 948 & 64 & 884 & 0.894 \\
\hline $\begin{array}{l}\text { Sobrepeso } \\
\left(25-29.9 \mathrm{~kg} / \mathrm{m}^{2}\right)\end{array}$ & 338 & 23 & 315 & 0.92 \\
\hline $\begin{array}{l}\text { Obesidad grado } 1 \\
\left(30-34.5 \mathrm{~kg} / \mathrm{m}^{2}\right)\end{array}$ & 119 & 8 & 111 & 0.986 \\
\hline $\begin{array}{l}\text { Obesidad grado } 2 \\
\left(35-39.9 \mathrm{~kg} / \mathrm{m}^{2}\right)\end{array}$ & 26 & 0 & 26 & \\
\hline $\begin{array}{l}\text { Obesidad grado } 3 \\
\left(>40 \mathrm{~kg} / \mathrm{m}^{2}\right)\end{array}$ & 8 & 2 & 6 & 0.059 \\
\hline
\end{tabular}

Fuente: Proyecto UP AMIGOS, 2008 (Una investigación multidisciplinaria en obesidad y factores socioambientales)

[1] Clasificación del IMC de acuerdo al criterio de la OMS, 2015.

Con la finalidad de caracterizar la calidad de la dieta de los aspirantes se evaluó el consumo de alimentos de acuerdo con el índice de alimentación saludable (Tabla 2).

\begin{tabular}{|c|c|c|c|c|c|}
\hline Variables & $\begin{array}{c}\text { Nunca o } \\
\text { casi nunca } \\
\mathrm{N}(\%)\end{array}$ & $\begin{array}{c}\text { Menos de } 1 \text { vez } \\
\text { por semana } \\
\mathrm{N}(\%)\end{array}$ & $\begin{array}{c}102 \text { veces por } \\
\text { semana } \\
\mathrm{N}(\%)\end{array}$ & 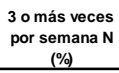 & $\begin{array}{l}\text { Diario } \\
\mathbf{N}(\%)\end{array}$ \\
\hline Cereales & $1(0.06)$ & $12(0.77)$ & $48(3.08)$ & $674(43.23)$ & 824 (52.85) \\
\hline Verduras & $43(2.76)$ & $270(17.32)$ & $442(28.35)$ & 695 (44.58) & 109 (6.99) \\
\hline Frutas & $18(1.15)$ & $128(8.21)$ & $344(22.07)$ & 738 (47.34) & $331(21.23)$ \\
\hline Leche y derivados & $11(0.71)$ & $66(4.23)$ & $166(10.65)$ & 679 (43.55) & $637(40.86)$ \\
\hline Carnes & $9(0.58)$ & $142(9.11)$ & $576(36.95)$ & 744 (47.72) & $88(5.64)$ \\
\hline Legumbres & $15(0.96)$ & $147(9.43)$ & $352(22.58)$ & 761 (48.81) & $284(18.22)$ \\
\hline Embutidos y fiambres & $37(2.37)$ & $242(15.52)$ & 517 (33.16) & $653(41.89)$ & $110(7.06)$ \\
\hline Dulces & $20(1.28)$ & $194(12.44)$ & 361 (23.16) & 649 (41.63) & 335 (21.49) \\
\hline Refresco con azúcar & $35(2.25)$ & $141(9.04)$ & $305(19.56)$ & $628(40.28)$ & $450(28.86)$ \\
\hline
\end{tabular}

Se encontró que, de todos los aspirantes, $54.95 \%$ "necesita cambios" (entre 50 y 80 puntos) en su dieta y $45.05 \%$ tiene una alimentación "poco saludable" ( $\leq$ 50 puntos). Como se puede observar en la Tabla 3 ningún aspirante tuvo una dieta saludable $(\geq 80$ puntos).

Sobre el riesgo de presentar depresión, se identificó que $6.68 \%$ de los aspirantes lo presentan (Tabla 3 ).

Tabla 3. Calidad de la dieta de acuerdo con el índice de alimentación saludable y el riesgo de presentar depresión en aspirantes universitarios, 2008

\begin{tabular}{lc}
\multicolumn{1}{c}{ Variables } & Población N (\%) \\
\hline \multicolumn{1}{c}{ Calidad de la dieta (IAS) } \\
Alimentación saludable & 0 \\
Necesita cambios & $855(54.95)$ \\
Alimentación poco saludable & $701(45.05)$ \\
\hline \multicolumn{2}{c}{ Riesgo de presentar depresión (CESD-10) } \\
Riesgo de depresión & $104(6.68)$ \\
Sin riesgo de depresión & $1452(93.32)$ \\
\hline \multicolumn{2}{r}{ Fuente: Proyecto UP AMIGOS, 2008 (Una investigación multidisciplinaria en } \\
\multicolumn{2}{r}{ obesidad y factores socio-ambientales). }
\end{tabular}

Se encontró que ser mujer tiene casi 3 veces más de presentar riesgo de depresión ( $\mathrm{RM}=2.783$, IC95\% 1.754-4.415) (Tabla 1).

En relación con el índice de alimentación saludable (IAS) y el riesgo de presentar depresión, no se encontraron diferencias significativas $(\mathrm{RM}=1.09$, IC95\% 0.735-1.63). Sin embargo, al momento de relacionar el consumo de alimentos con el riesgo de presentar depresión con cada una de las opciones del IAS, se encontró significancia estadística en dos grupos. Los aspirantes que consumían menos de 1 vez por semana carnes aumentaron en 1.5 el riesgo de presentar depresión (RM=1.67, IC95\% 1.112.50). En cuanto al grupo de embutidos, se observa que los participantes que consumen 3 o más veces 
por semana, pero no diario tienen casi 2 veces más riesgo de presentar depresión. (RM=1.90, IC95\% 1.19-3.04) (Tabla 4).

Tabla 4. Grupo de alimentos relacionados al riesgo de presentar depresión en aspirantes universitarios, 2008

\begin{tabular}{|c|c|c|c|}
\hline Alimentos & $R M^{*}$ & $\begin{array}{c}95 \% \text { intervalo } \\
\text { de confianza }\end{array}$ & Valor de P \\
\hline \multicolumn{4}{|l|}{ Cereales } \\
\hline 3 o más veces por semana, pero no diario & 1.27 & $0.16-9.94$ & 0.819 \\
\hline 1 o 2 veces por semana & 1.65 & $0.64-4.27$ & 0.298 \\
\hline Menos de 1 vez por semana & 0.7 & $0.46-1.07$ & 0.103 \\
\hline Nunca o casi nunca & 1.28 & $0.86-1.92$ & 0.219 \\
\hline \multicolumn{4}{|l|}{ Frutas } \\
\hline 3 o más veces por semana, pero no diario & 1.06 & $0.52-2.15$ & 0.87 \\
\hline 102 veces por semana & 1 & $0.619-1.61$ & 0.998 \\
\hline Menos de 1 vez por semana & 0.91 & $0.61-1.36$ & 0.656 \\
\hline Nunca o casi nunca & 1.05 & $0.65-1.71$ & 0.815 \\
\hline \multicolumn{4}{|l|}{ Verduras } \\
\hline 3 o más veces por semana, pero no diario & 0.93 & $0.54-1.59$ & 0.793 \\
\hline 1 o 2 veces por semana & 1.02 & $0.66-1.59$ & 0.906 \\
\hline Menos de 1 vez por semana & 0.98 & $0.65-1.46$ & 0.937 \\
\hline Nunca o casi nunca & 1.11 & $0.527-2.35$ & 0.776 \\
\hline \multicolumn{4}{|l|}{ Leche y derivados } \\
\hline 3 o más veces por semana, pero no diario & 0.66 & $0.20-2.15$ & 0.498 \\
\hline 102 veces por semana & 1.45 & $0.81-2.57$ & 0.202 \\
\hline Menos de 1 vez por semana & 1.16 & $0.78-1.73$ & 0.459 \\
\hline Nunca o casi nunca & 0.78 & $0.52-1.19$ & 0.262 \\
\hline \multicolumn{4}{|l|}{ Carnes } \\
\hline 3 o más veces por semana, pero no diario & 0.94 & $0.46-1.92$ & 0.881 \\
\hline 102 veces por semana & 0.51 & $0.32-0.82$ & 0.005 \\
\hline Menos de 1 vez por semana & 1.67 & $1.11-2.50$ & 0.013 \\
\hline Nunca o casi nunca & 1.42 & $0.67-3.04$ & 0.354 \\
\hline \multicolumn{4}{|l|}{ Legumbres } \\
\hline 3 o más veces por semana, pero no diario & 0.46 & $0.18-1.16$ & 0.102 \\
\hline 1 o 2 veces por semana & 0.96 & $0.60-1.56$ & 0.898 \\
\hline Menos de 1 vez por semana & 0.96 & $0.64-1.44$ & 0.871 \\
\hline Nunca o casi nunca & 1.3 & $0.80-2.11$ & 0.275 \\
\hline \multicolumn{4}{|l|}{ Embutidos } \\
\hline 3 o más veces por semana, pero no diario & 1.9 & $1.19-3.04$ & 0.008 \\
\hline 1 o 2 veces por semana & 0.76 & $0.49-1.19$ & 0.238 \\
\hline Menos de 1 vez por semana & 0.75 & $0.49-1.14$ & 0.181 \\
\hline Nunca o casi nunca & 1.26 & $0.62-2.58$ & 0.515 \\
\hline \multicolumn{4}{|l|}{ Dulces } \\
\hline 3 o más veces por semana, pero no diario & 1.09 & $0.61-1.97$ & 0.751 \\
\hline 102 veces por semana & 0.77 & $0.47-1.28$ & 0.329 \\
\hline Menos de 1 vez por semana & 0.9 & $0.60-1.36$ & 0.644 \\
\hline Nunca o casi nunca & 1.44 & $0.92-2.26$ & 0.104 \\
\hline \multicolumn{4}{|l|}{ Refresco con azúcar } \\
\hline 3 o más veces por semana, pero no diario & 0.95 & $0.47-1.96$ & 0.899 \\
\hline 102 veces por semana & 1.11 & $0.68-1.82$ & 0.654 \\
\hline Menos de 1 vez por semana & 1.13 & $0.76-1.69$ & 0.532 \\
\hline Nunca o casi nunca & 0.76 & $0.48-1.21$ & 0.257 \\
\hline
\end{tabular}

\section{Discusión}

Los estudiantes universitarios modifican sus patrones de alimentación en función de diversos factores entre ellos el propio contexto social, económico y ambiental que se vive durante la etapa universitaria (Contreras-Landgrave et al., 2013). Patrones de alimentación caracterizados por el consumo de dulces, galletas, snacks, comida rápida y por la ingesta disminuida de frutas/verduras y carnes se ha relacionado con el riesgo de presentar depresión en este grupo de edad (El Ansari et al., 2014). El presente estudio permitió identificar que existe una relación entre el alto consumo de embutidos, un bajo consumo de carne y el riesgo de presentar depresión.

En nuestra muestra de estudio, el patrón de alimentos estuvo caracterizado por un consumo elevado de sal proveniente de embutidos, alto consumo de frutas, verduras y cereales además de un elevado consumo de azúcar (refrescos y dulces). El patrón de alimentación elevado en embutidos, concuerda con el encontrado por Ortiz-Moncada, et al. (2012) en su estudio transversal en donde el patrón de alimentación de estudiantes españoles de los dos primeros ciclos de ingreso a la universidad estaba compuesto por un consumo excesivo de embutidos.

A su vez, existen estudios transversales que muestran que el tipo de dieta occidental constituido por carnes procesadas y azúcar es asociado a mayor predisposición a síntomas y trastornos psicológicos (Jacka et al., 2010; Oddy et al. 2009). Tales resultados son consistentes con el estudio de cohorte por Akbaraly et al. (2009) en donde encontraron una relación positiva entre el patrón alimentario de "alimentos procesados" caracterizado por un alto consumo de comida frita, carnes procesadas, cereales refinados, postres azucarados y lácteos ricos en grasa y puntuaciones más altas en el cuestionario Center for Epidemiological Studies Depression Scale (CESD.) Así mismo se informan asociaciones significativas entre la reducción de alimentos considerados como comida rápida o dietas consideradas menos saludables y una menor probabilidad de presentar síntomas depresivos (Liu et al., 2007; Sánchez-Villegas et al., 2011).

En relación al consumo de embutidos y carne, en nuestro estudio, el alto consumo de embutidos y el bajo consumo de carne se asociaron positivamente 
con el riesgo de depresión. El 41.89\% de la población consumía de 3 a más veces a la semana, pero no diario embutidos. Como primer punto, al documentar el alto consumo de embutidos en la población mexicana, se conoce que en México según datos de un estudio realizado por la Procuraduría Federal del Consumidor (PROFECO) (2010) el 36\% de los mexicanos consumen salchichas hasta tres veces por semana. De la misma forma en el estudio se confirma que el contenido de sodio de las salchichas es alto hasta 1.184 gramos-, por lo que las salchichas deben considerarse un alimento ocasional. En cuanto a los universitarios Oliveras-López et al. (2006), SangHee \& Ho- Kyung (2015) y Pérez-Correa et al. (2009) han descrito cómo son los hábitos alimentarios, se señala que en este grupo de edad es elevado el consumo de alimentos con alto contenido de sal.

Investigaciones sobre la relación entre el consumo de sal proveniente de alimentos aislados y el riesgo de presentar depresión son pocos y nulos aquellos que se lleven a cabo en estudiantes universitarios. Sin embargo, si se toma en consideración que los embutidos son alimentos que están presentes en aquellas preparaciones consideradas como comida rápida, existe evidencia de su relación con el riesgo de presentar depresión; como se señala en el estudio de cohorte por Sánchez-Villegas et al. (2011) donde encontraron una relación dosis-respuesta positiva en población española entre el consumo de comida rápida (hamburguesas, salchichas, pizza) y el riesgo de depresión.

Se ha documentado que la relación de este tipo de patrones de alimentos ricos en ácidos grasos trans ha sido determinada como un factor que aumenta el riesgo de padecer esta enfermedad mental (Liu et al., 2007; Sánchez-Villegas et al., 2011). Se han encontrado concordancias entre la patología de la depresión y la enfermedad cardiovascular, y esto es debido a que los mediadores de los efectos adversos de los ácidos grasos trans en la enfermedad cardiovascular incluyen aumento en las concentraciones plasmáticas de lipoproteínas de baja densidad, reducciones en lipoproteínas de alta densidad, colesterol, cambios proinflamatorios y disfunción endotelial. Debido a que la depresión también se asocia con un estado de inflamación de bajo grado, disfunción endotelial e inadecuados perfiles de lípidos, las modificaciones biológicas causadas por este tipo de ácidos grasos también podrían ser responsables de efectos perjudiciales en la depresión (Sánchez-Villegas \& MartínezGonzález, 2013).

En relación a los resultados con el consumo de carne, existe un aumento en el riesgo de presentar depresión al consumir menos de $1 \mathrm{vez}$ por semana ( $\mathrm{RM}=1.67$, IC95\% 1.11-2.50). Ante estos resultados la literatura marca observaciones diferentes. Existen estudios que comprueban que el consumo de carne no se asocia con depresión (Miyake, et al., 2013; Kim, Choi, Lee \& Park, 2015). No obstante, la población en la que se realizaron las investigaciones incluía adolescentes y embarazadas, siendo que las condiciones de estos estados de vida son diferentes al de la población de este estudio, además algunos factores de confusión pudieran haber mermado la asociación entre el consumo de carne y depresión (Yi Zhang et al., 2017). El bajo consumo de carne tal vez se relacione con el mayor consumo de otros alimentos como vegetales y frutas, teniendo en consideración que estos grupos de alimentos tienen efectos beneficiosos ante el riesgo de presentar depresión (SánchezVillegas et al., 2009).

Al enfocarnos solamente en este alimento, específicamente en la vitamina B12. Existen varios estudios que concuerdan con nuestros resultados (Baines, Poers \& Brown, 2006) como, por ejemplo, el estudio llevado a cabo en estudiantes vegetarianos donde se relaciona la deficiencia de la vitamina B12 con mayor probabilidad de presentar desordenes depresivos (Larsson, Clock, Nordrehaug-Astrom, Haugerjorden y Johansson, 2002). Debido a que se tiene por principal y única fuente de esta vitamina los productos de origen animal. Esto podría explicarse por el posible mecanismo del efecto biológico de esta vitamina y su influencia en los procesos cerebrales (Michalak, Zhang y Jacobi, 2012). Este nutriente es esencial para el sistema nervioso central y puede modular el ánimo a través de varios mecanismos que son necesarios en la síntesis del metabolismo de la serotonina y otros neurotransmisores (Rodríguez \& Solano, 2008).

Por otro lado, nuestros resultados concuerdan con los de Mikolajczyk et al. (2009) en su estudio transversal donde concluyeron que, en estudiantes de primer grado, existe una relación entre el bajo consumo de carne y el riesgo de presentar depresión. 
Al igual que ellos, nosotros no tenemos una interpretación clara de este hallazgo. Debido que al ser un estudio transversal no podemos visualizar de forma adecuada si el riesgo de la enfermedad es producto del bajo consumo de carne o viceversa.

En este trabajo se encontró también una relación significativa entre ser mujer y el riesgo de presentar depresión, hallazgo que coincide con lo descrito por la literatura (Mikolajczyk et al., 2008). Essau, Lewinsohn, Seeley y Sasagawa (2010) refieren que los factores biológicos que están asociados con mayor frecuencia de depresión en mujeres son cambios hormonales (DeRose, Wright y BrooksGunn, 2006), factores sociales como adversidad en la infancia (Daley, Hammen y Rao, 2000), factores cognitivos como la tendencia a la rumiación (NolenHoeksema, 2001) y factores psicológicos como la experiencia (Shih, Eberhart, Hammen y Brennan, 2006). En relación con los factores biológicos, en cuanto a los cambios hormonales, se ha visto que influyen junto con otros factores en el desarrollo de la depresión (Jacka \& Berk, 2007).

Específicamente en cuanto a la alimentación podríamos argumentar que la dieta modula algunos factores biológicos, dando como resultado un impacto en el desarrollo o en el curso de esta enfermedad mental (Jacka \& Berk, 2007). Se ha observado que el consumo de patrones de alimentos constituidos por comida rica en azúcares y grasas como dulces, galletas, snacks y comida rápida en mujeres se utiliza para mejorar estados de ánimo desagradables, a través del aumento de la serotonina (Yanovski, 2003); considerando que la depresión se ha asociado con bajos niveles de este neurotransmisor (Toker at al., 2010).

De igual manera Rivera-Rivera, Rivera-Hernández, Pérez-Amezcua, Leyva-López \& de Castro (2015) explican que esta asociación podría ser debido a que las expectativas de rol de género y estructura familiar desempeñan un papel importante, no sólo en la conducta del adolescente sino también en su salud mental aparte de los factores genéticos, hormonales y culturales.

Entre las limitaciones de este estudio se encuentra el tipo de alimentos que se incluyeron dentro de los grupos utilizados en el índice de alimentación saludable, ya que a pesar de que se identificaron alimentos que consume la población mexicana en general, no todos se adaptaban a la misma y con menos certeza a los alimentos de la región. Se han adaptado los alimentos del índice según la alimentación en algunas regiones, es el caso de un estudio llevado a cabo en Tabasco en donde adaptaron los alimentos de la región sureste del país (Muñoz-Cano, Cordova-Hernandez y ValleLeveaga, 2015). Además, el diseño como tal del estudio es una limitante; debido a que al ser de tipo transversal no se puede diferenciar en qué medida los patrones de alimentos son un factor de riesgo o una consecuencia. Otro punto por considerar como posible debilidad es el tiempo transcurrido desde el levantamiento de datos hasta su análisis que fue aproximadamente de ocho años.

\section{Conclusiones}

La relevancia de esta investigación radicó en que se obtuvo evidencia de importantes relaciones de los alimentos con el riesgo de presentar depresión en los aspirantes universitarios, así como la descripción de sus patrones de alimentación, siendo que es una población vulnerable a las enfermedades físicas y mentales debido a las etapas de cambio que se viven tanto sociales, familiares y académicas. Esta información permite centrar la atención en aquellos alimentos que los hacen vulnerables a este tipo de padecimientos. Se pueden realizar futuras investigaciones que complementen el presente estudio, con el fin de determinar el comportamiento de esta problemática a lo largo de la realización de los estudios académicos.

\section{Bibliografía}

Álvarez Munárriz, L. \& Álvarez de Luis A. (2009). Estilos de vida y alimentación. Gazeta de Antropología, 25(1), 1-14. Recuperado de: http://www.gazetaantropologia.es/?p=1916

Álvarez Gasca, M. A., Guillén Cadena, D. M., Ríos Saldaña, M. R. \& Jiménez Martínez, C. A. (2011). Evaluación del estilo de vida y depresión en estudiantes. Desarrollo Cientif Enferm 19(8), 264-268. Recuperado de: http://www.indexf.com/dce/19pdf/19-264.pdf

Akbaraly, T. N., Brunner, E. J., Ferrie, J. E., Marmot, M. G., Kivimaki, M. \& Singh- Manoux, A. (2009). Dietary pattern and depressive symptoms in middle age. Br J Psychiatry, 195, 408-413. Recuperado de: https://www.ncbi.nlm.nih.gov/pmc/articles/PMC2801 $825 /$ 
Appleton, K. M., Woodside, J. V., Yarnell, J. W., Arveiler, D., Haas, B., Amouyel, P., ... Evans A. (2007). Depressed mood and dietary fish intake: direct relationship or indirect relationship as a result of diet and lifestyle?.J Affect Disord, 104(1-3), 217-23. Recuperado http://dx.doi.org/10.1016/j.jad.2007.03.012

Arrivillaga-Quintero, M., Cortés-García, C., GoicocheaJiménez, V. \& Lozano-Ortiz, T. (2004). Caracterización de la depresión en jóvenes universitarios. Universitas Psychologica, 3 (1), 17-26. Recuperado http://www.redalyc.org/articulo.oa?id=64730103

Baines, S., Poers, J. \& Brown, W. J. (2006). How does the health and well-being of young Australian vegetarian and semi-vegetarian women compare with nonvegetarians? Public Health Nutrition, 10, 436-442. Recuperado https://doi.org/10.1017/S1368980007217938

Bojorquez Chapela, I. \& Salgado de Snyder, N. (2009). Psychometric characteristics of the Center for Epidemiological Studies-Depression Scale (CES-D), 20- and 10-item versions, in women from a Mexican rural area. Salud Mental, 33, 299-307. Recuperado de: http://www.scielo.org.mx/scielo.php?pid=S0185$\underline{33252009000400005 \& \text { script=sci_abstract }}$

Contreras-Landgrave, P. G., Camacho-Ruiz, E. J., IbarraEspinosa, M. L., López-Gutierréz, L. R., Escoto-Ponce de León, M. C., Pereira-Abagaro, C. ... MunguíaOcampo, L. V. (2013). Los hábitos alimentarios de estudiantes universitarios. Red digital universitaria,14. Recuperado http://www.revista.unam.mx/vol.14/num11/art48/

Daley, S. E., Hammen, C. \& Rao, U. (2000). Predictors of first onset and recurrence of major depression in young women during the 5 years following high school graduation. J Abnorm Psychol, 109, 525-533. Recuperado de: http://psycnet.apa.org/record/2000$\underline{05424-018}$

DeRose, L. M., Wright, A. J. \& Brooks-Gunn, J. (2006). Does puberty account for the gender differential in depression? En: Keyes CLM, Goodman SH (eds.). Women and depression, 89-128. New York: Cambridge University Press.

El Ansari, W., Adetunji, H. \& Oskrochi, R. (2014). Food and mental health: relationship between food and perceived stress and depressive symptoms among university students in the United Kingdom. Cent Eur J Public Health, 22, 90-97. Recuperado de: http://apps.szu.cz/svi/cejph/archiv/2014-2-04-full.pdf
Essau, C. A., Lewinsohn, P. M., Seeley, J. R. \& Sasagawa, S. (2010). Gender differences in the developmental course of depression. J Affect Disord, 127, 185-190. Recuperado

de: https://www.ncbi.nlm.nih.gov/pmc/articles/PMC3754 $\underline{427 /}$

Fouilloux-Morales, C., Barragán-Pérez, V., Ortiz-León, S., Jaimes-Medrano, A., Urrutia Aguilar, M. E. \& Guevara-Guzmán, R. (2013). Síntomas depresivos y rendimiento escolar en estudiantes de Medicina. Salud mental, 36(1), 59-65. Recuperado de http://www.scielo.org.mx/scielo.php?script=sci arttex t\&pid=S0185-33252013000100008\&lng=es\&tlng=es

Hakkarainen, R., Partonen, T., Haukka J., Virtamo, J., Albanes, D., Lönnqvist, J. (2004). Is low dietary intake of omega-3 fatty acids associated with depression?. Am J Psychiatry 161(3), 567-9. Recuperado de: https://doi.org/10.1176/appi.ajp.161.3.567

Hysenbegasi, A., Hass, S. L., Rowland, C. R. (2005). The Impact of Depression on the Academic Productivity of University Students. J Ment Health Policy Econ 8, 145151. Recuperado de: http://www.icmpe.net/fulltext.php?volume $=8 \&$ page $=$ $145 \&$ year $=2005 \&$ num $=3 \&$ name $=$ Hysenbegasi $\% 20 \mathrm{~A}$

Jacka, F. N. \& Berk, M. (2007). Food for thought. Acta Neuropsychiatrica, $\quad 19, \quad 321-323$ https://doi.org/10.1111/j.1601-5215.2007.00246.x

Jacka, F. N., Pasco, J. A., Mykletun, A., Williams, L. J., Hodge, A. M., ... Berk, M. (2010). Association of Western and Traditional Diets With Depression and Anxiety in Women. Am J Psychiatry, 167(3), 305-311. doi: 10.1176/appi.ajp.2009.09060881

Kennedy, E. T., Ohls, J., Carlson, S. \& Fleming, K. (1995). The Healthy Eating Index: design and applications. J Am Diet Assoc, 95(10), 1103-8. DOI: 10.1016/S00028223(95)00300-2

Kim, T. H., Choi, J. Y., Lee, H. H., Park, Y. (2015). Associations between dietary pattern and depression in Korean adolescent girls. J Pediatr Adolesc Gynecol, 28(6), 533-7. DOI: 10.1016/j.jpag.2015.04.005,

Kohout, F. J., Berkman, L. F., Evans, D. A. \& CornoniHuntley, J. (1993). Two shorter forms of the CES-D depression symptoms index. Journal of Aging and Health, 5, 179-193.Recuperado de: http://journals.sagepub.com/doi/abs/10.1177/0898264 39300500202?url_ver=Z39.882003\&rfr_id=ori\%3Arid\%3Acrossref.org\&rfr_dat=cr pub\%3Dpubmed\& 
Larsson, C. L., Clock, K. S., Nordrehaug-Astrom, A., Haugerjorden, O. \& Johansson, G. (2002). Lifestylerelated characteristics of young low-meat consumers and omnivores in Sweden and Normay. Journal of Adolescent Health, 31, 190-198. Recuperado de: http://dx.doi.org/10.1016/S1054-139X(02)00344-0

Liu, C., Xie, B., Chou, C. P., Koprowski, C., Zhou, D., Palmer, P., ...Anderson Johnson, C. (2007). Perceived stress, depression and food consumption frequency in the college students of china seven cities. Physiol Behavior, 92(4), 748-754. Recuperado de: https://www.ncbi.nlm.nih.gov/pubmed/17585967

Major Depression. (noviembre, 2017). National Institute of Mental Health. Recuperado de: https://www.nimh.nih.gov/health/statistics/majordepression.shtml.

Martínez-González, M. A. \& Sánchez-Villegas, A. (2016). Conference on 'Nutrition at key life stages: new findings, new approaches' Symposium 1: Nutritional issues in adolescence and adulthood. Food patterns and the prevention of depression. Proceedings of the Nutrition Society, 75, 139-146. doi:10.1017/S0029665116000045

Meyer, B. J., Kolanu, N., Griffiths, D. A., Grounds, B., Howe, P. R., Kreis, I. A. (2013). Food groups and fatty acids associated with self-reported depression: an analysis from the Australian National Nutrition and Health Surveys. Nutrition, 29(7-8), 1042-7. doi: 10.1016/j.nut.2013.02.006.

Michalak, J., Zhang, X. C. \& Jacobi, F. (2012). Vegetarian diet and mental Disorders: results from a representative community survey. Int J Behav Nutr Phys Act, 9, 67. Recuperado https://www.ncbi.nlm.nih.gov/pmc/articles/PMC3466 $124 /$

Mikolajczyk, R.T., Maxwell, A. E., El Ansari, W., Naydenova, V., Stock, C., Ilieva, S.... Nagyova, I. (2008). Prevalence of depressive symptoms in university students from Germany, Denmark, Poland and Bulgaria. Soc Psychiatry Psychiatr Epidemiol, 43, 105-112. Recuperado de: https://link.springer.com/article/10.1007\%2Fs00127007-0282-0

Mikolajczyk, R. T., El Ansari, W. \& Maxwell, A. E. (2009). Food consumption frequency and perceived stress and depressive symptoms among students in three European countries. Nutr J, 8:31. Recuperado de: https://www.ncbi.nlm.nih.gov/pmc/articles/PMC2716 $\underline{364 l}$
Miyake, Y., Tanaka, K., Okubo, H., Sasaki, S. \& Arakawa, M. (2013). Fish and fat intake and prevalence of depressive symptoms during pregnancy in Japan: baseline data from the Kyushu Okinawa maternal and child health study. J Psychiatr Res, 47(5),572-8. DOI: 10.1016/j.jpsychires.2013.01.012

Muñoz-Cano, J. M., Cordova-Hernandez, J. A. \& ValleLeveaga, D. (2015). El índice de alimentación saludable de estudiantes de nuevo ingreso a una universidad de México. Nutr Hosp, 31, 1582-1588. Recuperado http://www.redalyc.org/pdf/3092/309238513017 pdf

Navarro-Norte, A. \& Moncada-Ortiz, R. (2011). Calidad de la dieta española según el índice de alimentación saludable. Nutr Hosp, 26, 330-336.Recuperado de: http://scielo.isciii.es/pdf/nh/v26n2/14_original_07.pdf

Nolen-Hoeksema, S. (2001). Gender differences in depression. Current directions. Psychol Science, 10, 173-176. Recuperado de: http://bip.rcpsych.org/content/177/6/486.long

Oddy, W. H., Robinson, M., Ambrosini, G. L., O’Sullivan, T. A, de Klerk, N. H., Beilin, L. J., ... Stanley, F. J. (2009). The association between dietary patterns and mental health in early adolescence. Prev Med, 49, 39$44 . \quad$ Recuperado de: https://www.sciencedirect.com/science/article/pii/S00 91743509002643?via\%3Dihub

Oliveras- López, M. J., Nieto-Guindo, P., Agudo-Aponte, E., Martínez-Martínez, F., López-García de la Serrana \& López Martínez, M.C. (2006). Evaluación nutricional de una población universitaria. Nutr Hosp, 21, 179-83. Recuperado de: http://scielo.isciii.es/pdf/nh/v21n2/original8.pdf

Olvera-Castillo, M. C., Palos-Lucio, A. G., AradillasGarcía, C \& Padrón-Salas, A. (2018). Relación entre el índice de masa corporal con la ingesta de frutas, verduras y la realización de actividad física. Tlatemoani, 27, 110-130. Recuperado de: https://www.eumed.net/rev/tlatemoani/27/sobrepeso.h tmlOrganización Mundial de la Salud (OMS). (2015): Obesidad y sobrepeso. Disponible en: http://www.who.int/mediacentre/factsheets/fs311/es/. Consultado en 29/05/2017 a las 23:17

Ortiz-Moncada, R., Norte-Navarro, A. I., Zaragoza-Marti, A., Fernández-Sáez, J. \& Davó Blanes, M. C. (2012). ¿Siguen patrones de dieta mediterránea los universitarios españoles? Nutr Hosp, 27, 1952-1959. Recuperado http://dx.doi.org/10.3305/nh.2012.27.6.6091 
Pérez-Correa, N. G., Moya de Sifontes, M. Z., Bauce, G., Cueva, E., Peña, R., Flores, Z.... García, P. (2009). Patrones y hábitos alimentarios: reflejo de lo que comen los jóvenes ucevistas. Revista de la Facultad de Medicina, 32, 67-74. Recuperado de: http://www.scielo.org.ve/scielo.php?pid=S079804692009000100011\&script=sci_abstract

Procuraduría Federal del Consumidor (PROFECO). (2010). Revista del consumidor analiza la calidad de 62 marcas de salchichas tipo viena y de pavo. Recuperado de:

http://www.profeco.gob.mx/prensa/prensa10/septiem bre10/bol113.asp

Raffaelli, M., Drumond- Andrade, F. C., Poppe, E. M., Sanchez-Armass, O., Vazquez-Vidal, I. \& AradillasGarcía, C. (2013). Gender and Age-Related Differences in Depressive Symptoms and Health Behaviors among Mexican Youth. R. Interam. Psicol, 47 (1), 83-90. Recuperado de: http://www.redalyc.org/articulo.oa?id=28426980010

Riveros, Q. M., Hernández, V. H. \& Rivera, B. J. (2014). Niveles de depresión y ansiedad en estudiantes universitarios de Lima Metropolitana. Revista de Investigación en Psicología, 10(1), 91-102.Recuperado de: http://dx.doi.org/10.15381/rinvp.v10i1.3909

Rivera-Rivera, L., Rivera-Hernández P., Pérez-Amezcua, B., Leyva-López, A. \& de Castro, F. (2015). Factores individuales y familiares asociados con sintomatología depresiva en adolescentes de escuelas públicas de México. Salud Pública de México, 57, 219-226. Recuperado http://www.scielo. org mx/scielo.php?script=sci arttex t\&pid=S0036-36342015000300010

Rodríguez, A. \& Solano, M. (2008). Nutrición y Salud Mental: Revisión Bibliográfica. Rev Post Psiquiat UNAH, 1(3),1-5. Recuperado de: http://www.bvs.hn/RHPP/pdf/2008/pdf/Vol1-3-20083.pdf

Sánchez-Villegas, A., Delgado-Rodríguez, M., Alonso, A., Schlatter, J., Lahortiga, F., Serra Majem, L... Martínez-González, M. A. (2009). Association of the Mediterranean dietary pattern with the incidence of depression: the Seguimiento Universidad de Navarra/ University of Navarra follow-up (SUN) cohort. Arch Gen Psychiatry, $66 \quad$ (10),10908.DOI:10.1001/archgenpsychiatry.2009.129

Sánchez-Villegas, A., Toledo, E., Irala, J., Ruiz-Canela, M., Pla-Vidal, J. \& Martínez-González, M. (2011). Fast-food and commercial baked goods consumption and the risk of depression. Public Health Nutr, 15, 424432.Recuperado https://doi.org/10.1017/S1368980011001856

Sánchez-Villegas, A. \& Martínez-González, M. A. (2013). Diet, a new target to prevent depression?. BMC Medicine 11:3. Recuperado de: https://doi.org/10.1186/1741-7015-11-3

Sang-Hee, L. \& Ho- Kyung, R. (2015). A Comparative Analysis of Salt-Related Dietary Patterns According to the Sodium Intake of College Students in Busan.The Korean Journal of Community Living Science, 26, 167-176. Recuperado de: http://dx.doi.org/10.7856/kjcls.2015.26.1.167

Shih, J. H., Eberhart, N. K., Hammen, C. L. \& Brennan, P. A. (2006). Differential exposure and reactivity to interpersonal stress predict sex differences in adolescent depression. J Clin Child Adolesc Psychol, 35, 103-115. Recuperado de: http://dx.doi.org/10.7856/kjcls.2015.26.1.167

Toker, L., Amar, S., Bersudsky, Y., Benjamin, J. \& Klein, E. The biology of tryptophandepletion and mood disorders. (2010). Isr J Psychiatry Relat Sci, 47, 46-55. Recuperado https://www.ncbi.nlm.nih.gov/pubmed/20686199

Wiley, A. R., Flood, T. L., Andrade, F. C. D., Aradillas, C. \& Cerda, E. (2011). Family and Individual Predictors of Physical Activity for Older Mexican Adolescents. Journal of Adolescent Health, 49, 222-224. Recuperado de: DOI: 10.1016/j.jadohealth.2010.11.258.

Yanovski, S. Sugar and fat: cravings and aversions. (2003). J Nutr, 133, 835S-837S. https://doi.org/10.1093/jn/133.3.835S

Zhang, Y., Yang, Y., Xie, M., Ding, X., Li, H., Liu, Z., Peng, S. (2017). Is meat consumption associated with depression? A meta-analysis of observational studies. BMC Psychiatry, 17, 409. DOI 10.1186/s12888-017$1540-7$ 